\title{
Review: Psychological Problems of Pregnant Women and Progress of Psychological Guidance in Recurrent Miscarriage
}

\author{
Haiyan Lin ${ }^{1}$, Chenyun $\mathrm{Xu}^{2 *}$ \\ ${ }^{1}$ The Fourth People's Hospital of Haikou, Haikou, China \\ ${ }^{2}$ Hainan General Hospital, Haikou, China \\ Email: ^g2002m@163.com
}

How to cite this paper: Lin, H.Y. and $\mathrm{Xu}$, C.Y. (2021) Review: Psychological Problems of Pregnant Women and Progress of Psychological Guidance in Recurrent Miscarriage. Health, 13, 729-735.

https://doi.org/10.4236/health.2021.137056

Received: June 21, 2021

Accepted: July 11, 2021

Published: July 14, 2021

Copyright (อ 2021 by author(s) and Scientific Research Publishing Inc. This work is licensed under the Creative Commons Attribution International License (CC BY 4.0).

http://creativecommons.org/licenses/by/4.0/

\section{(c) (i) Open Access}

\begin{abstract}
In order to understand the psychological problems of pregnant women, this article explores the psychological problems and the sources of psychological stress of pregnant women who have a history of recurrent miscarriage after pregnancy. The analysis shows that the psychological stress of pregnant women may be one of the reasons for recurrent miscarriage. Strengthening mental health education during pregnancy can avoid the stimulation of bad emotions and environment, prevent the spontaneous abortion caused by psychological reasons, and improve the quality of pregnancy. It is particularly important to grasp the mental state of pregnant women with recurrent abortion timely and carry out corresponding psychological counseling and psychological nursing.
\end{abstract}

\section{Keywords}

Pregnant Women, Recurrent Abortion, Psychological Factors, Psychological Guidance

\section{Introduction}

The probability of one abortion during pregnancy is $15 \%-20 \%$, and $1 \%-5 \%$ pregnant women have recurrent spontaneous abortion (RSA), of which $40 \%$ $55 \%$ have no clear cause at present [1]. Pregnancy itself is a complex psychological process, and there are many factors to form the source of stress, which results in highly maternal stress state, causing many of the psychological barriers. However, the current research on the pathogenesis and treatment of repeated abortion is mainly from the biological point of view, ignoring the psychosocial 
characteristics of patients with repeated abortion and the causes of abortion. There are few researches at home and abroad, and they are not systematic and comprehensive [2]. Recurrent abortion is a common disease in obstetrics department. Due to changes in environmental factors and social factors, spontaneous abortion has been on the rise in recent years [3]. More and more women have multiple spontaneous abortions. Due to repeated pregnancies, repeated abortions and treatment, both the couple and the couple have suffered great blows both mentally and economically [4]. Especially pregnant women themselves to bear physical trauma, but also to bear the pressure from family, relatives, friends, can be said to be a physical and mental damage. With the change of medical model, the mental health of pregnant women has been paid more and more attention. According to research reports, mental stimulation and poor mental health status of pregnant women have a certain relationship with congenital malformation, and it is of great significance to the health and safety of mother and child and the pregnancy outcome. Therefore, only by doing a good job of mental and physical comfort during pregnancy and cultivating their ability to vent and relax, can they maintain a good mental state [5]. So, patients with recurrent abortion have those psychological problems, and how to deal with pregnancy has attracted more attention from medical personnel. This paper gives some discussion on this aspect.

\section{Analysis of Psychological Problems of Pregnant Women with Recurrent Abortion}

\subsection{Tension and Anxiety}

Conception is a natural human instinct and right that cannot be arranged by itself. The rate of spontaneous abortion was $24 \%$ after 1 spontaneous abortion, $26 \%$ after 2 spontaneous abortion, and $32 \%$ after 3 spontaneous abortion [6]. So patients with recurrent spontaneous abortion are very worried about another abortion after conception. They want to be pregnant, and afraid of being pregnant, showing tension and anxiety before pregnancy, and worry and fear after pregnancy. According to this characteristic, medical staff should first stabilize their emotions, explain the consequences of excessive anxiety, and give them pre-pregnancy guidance. For example, once pregnancy is discovered, they should seek medical treatment as soon as possible, monitor in time, and use drugs rationally [7].

\subsection{Guilt and Unworthiness}

"Karma for SINS committed in previous lives" is deeply rooted in the minds of some people. They look coldly at, blame, and make their heart sad, so they feel self-reproach, guilt, and unworthiness. For this point of view, first of all, to guide the tree, the correct outlook on life, values, treat oneself check it, have a correct understanding of disease of superstition, forgive, for they create the opportunity to reduce pressure, and discuss the basic knowledge of infertility, answer various 
questions, introduce the same cases cured, relieve mental pressure [8].

\subsection{Depression and Sadness}

As a result of the influence of the old traditional concept, some people often put infertility of all the reasons to blame on the woman. The sarcasm of in-laws, the indifference of husbands and the sneers of neighbors cause the women's extreme psychological depression, causing them to be depressed, sad and endocrine disorders. Husbands can't understand their wives' feelings, and some of them are even faced with the trouble that their families may break up [9]. In the face of such a heavy blow, first of all, the woman should be provided with an appropriate opportunity to vent, and at the same time, the husband and his family should do their job well. The necessity of both husband and wife seeing a doctor at the same time should be emphasized, and the cooperation and communication between the couple should be induced in spirit to avoid mutual criticism, so as to ensure a smooth, accurate and rapid diagnosis and treatment.

\subsection{Loneliness and Annoyance}

Unable to have a healthy child, women's self-esteem is severely damaged, they have no interest in social activities, and refuse to socialize and interact with family. In view of this psychology, first of all, opportunities are created to communicate with them [10]. Pay special attention to the emotional communication with them, first of all to respect, care, equality, gentle attitude to be their friends, understand their hobbies, personality and habits, encourage the initiative to communicate with people, with parents, friends, relieve pressure, so that the lonely heart gradually into the society.

\subsection{Sexual Disturbance}

Especially for rural patients, the lack of sexual knowledge combined with the torture of frequent examinations, treatments and surgeries aggravates mental stress, leading to low sexual desire and sexual dysfunction in some couples [11]. Both sides have fear of sexual life, forming a vicious circle. In the face of this psychological state, take relaxation therapy, let them participate in sports and sports activities, do what they like to do, the attention from the pain or depression of the state of transfer, so that the psychological pressure can be effectively relieved, restore the normal state of life.

\section{The Source of Psychological Pressure in Pregnant Women with Recurrent Abortion}

\subsection{Their Fertility Requirements}

I want to have a healthy child of my own, and to prove that I am a normal human being and have normal fertility. Past misfortunes have placed all hope in this pregnancy, anxious for the healthy development of the fetus and worried 
about its ability to carry on. The psychological pressure aggravates, longs for treatment to be effective.

\subsection{Sympathy or Contempt from Relatives and Communities Around}

In many areas, especially in some rural areas, the idea of carrying on the family line is deeply rooted. Under the influence of traditional Chinese concepts and social customs, they are forced to bear the pressure from many people around them, especially from relatives and friends within the family, mainly from their spouses and parents. Studies have shown that the lower the mood of patients and family members, the greater the pressure patients bear [12]. This undoubtedly makes infertile patients bear a lot of pressure.

\subsection{Its Limited Bearing Capacity}

The limited psychological capacity of patients to their own disease is also a source of this pressure. The frequency, duration and etiology of abortion, family background and treatment process all affect the pressure suffered by patients, etc. It has been found that patients admitted that they were very nervous during the treatment process, even affecting sleep and diet [13]. Many pregnant women show increased stress and fear in the weeks after the last miscarriage; Fear that this pregnancy will have the same outcome as the last one.

\subsection{Economic Pressure}

Family living environment and economic sources of restrictions, make the pregnant women in the hospital for fetal treatment psychological production of a sense of guilt, for fear of this fetal failure. Money is wasted. Make the family that is not rich already worse.

\subsection{Other Factors}

Social support is an important factor affecting psychological problems during pregnancy. Wu Yuanping et al. [14] found in their survey that pregnant women's interpersonal sensitivity and anxiety are negatively correlated with social support, that is, the higher the level of social support, the more sensitive the interpersonal relationship is and the less anxiety symptoms are. The incidence of prenatal depression was significantly negatively correlated with emotional support, material support, information support and evaluation support during pregnancy.

\section{Conduct Psychological Guidance for Pregnant Women with Recurrent Abortion}

\subsection{Psychological Guidance of Health Education}

First of all, medical staff can respect and care for patients, and protect patients' self-esteem and privacy. In the treatment, nursing process to give spiritual com- 
fort, reduce their fear of pregnancy. To build a harmonious doctor-patient relationship, health education including carefully provide sufficient information for patients, to discuss their expectations, after and treatment on the disease development outlook, share the treatment may be forming pressure to them, to make patients in mental comfort and emotional stability [15]. For patients with recurrent abortion, due to repeated abortion and treatment, they lack confidence in this pregnancy and have heavy misgivings [16]. For its coke Liao, depression, pessimism and worry; Make friends with them talk, let them say psychological words, give more sympathy, support and encouragement, inform the patient about my section of the treatment of recurrent abortion and successful examples, in order to stabilize pregnant women's psychology, establish confidence. Use good language, sincere attitude, and avoid rigid preachy introduction. Make patients feel warm and secure from our language, behavior and behavior, so as to eliminate the discomfort brought by unfamiliar environment to patients. Through communication with patients, in order to understand the psychological state of patients in the shortest time, timely eliminate adverse psychological factors that are not conducive to pregnancy, understand and protect the privacy of patients, remove concerns, grasp the proper balance, create a relaxed and happy atmosphere, guide pregnant women to increase interest in activities.

\subsection{Measures to Relieve Psychological Pressure}

\subsubsection{Search for Information}

Correctly understand the impact of emotional changes on pregnancy, through understanding the relevant knowledge of the success of the treatment of recurrent abortion, introduce some patients with successful fetal treatment to know them, in order to achieve relaxation of mood, relieve psychological pressure [17]. Instruct patients to make the best use of their time by listening to music, exercising, or attending health lectures in the hospital. Make their attention from the pain or depression transfer, so that the psychological pressure has been effectively relieved. This helps to improve the patient's psychological tolerance to treatment and is more conducive to the coordination of treatment, thus improving the success rate of pregnancy [18].

\subsubsection{Psychological Counseling}

Due to the unique social and psychological characteristics of this patient group, the hospital actively suggests and implements psychological counseling or psychotherapy for patients. On the one hand, it can relieve the pressure of patients, improve their psychological tolerance to setbacks, provide coping skills to reduce emotional reactions and improve the family [19]. On the other hand, they should be provided with sufficient information and medical consultation to guide the procedure and timing of treatment.

\subsubsection{Other Measures}

Family support, research has shown that abortion patients at the time of pain rely mainly on their spouses and family support, bad social factors influence the 
patient's coping styles, to support its adverse emotional responses, so should to the social from all walks of life calls for changing the secular ideas, make society more tolerance and care for them to reduce their mental burden and the pressure of family and society [20].

\section{Conclusions}

With the rapid development of clinical psychology in recent years, psychology has greatly promoted the field of obstetrics and gynecology. The physiological changes during pregnancy will have a psychological impact on pregnant women. Symptoms result from physical and psychological changes in a pregnant woman during pregnancy. It is generally considered to be the normal psychological reaction of pregnant women. But pregnancy such as a long time or serious psychological changes, such as anxiety, depression, will increase the incidence of miscarriage, premature birth. Therefore, in order to cope with this change, we carry out psychological counseling for pregnant women with recurrent abortion, inform them of the adverse effects of excessive tension and anxiety on pregnan$\mathrm{cy}$, and guide pregnant women to actively cooperate with treatment and maintain a comfortable mood, which is beneficial.

During the growth and development of the embryo, it safely passed the dangerous period of pregnancy. Teach them relaxation techniques such as listening to music, walking, etc. We provide psychological comfort to pregnant women with recurrent abortion, and use our mature technology for the treatment of recurrent abortion to help patients relax and actively cooperate with treatment to achieve a good effect. The influence of psychosocial factors on habitual abortion must be paid more attention to by clinical staff. At the same time as physical treatment for women with habitual abortion, psychological health education should be carried out in families with history of habitual abortion and families about to give birth, and psychological support and help should be provided. Relieve their anxiety, depression, sensitive symptoms, so that the body and mind can be fully rested, to achieve the therapeutic effect.

\section{Foundation Project}

Hainan Health Commission Science and Technology Project (No. 20A200187).

\section{Conflicts of Interest}

The authors declare no conflicts of interest regarding the publication of this paper.

\section{References}

[1] Lin, Q.D. and Qiu, L.H. (2009) Epidemiology of Recurrent Spontaneous Abortion. 2nd Edition, People's Medical Publishing House, Beijing, 359-360.

[2] Bian, W.W., Yang, L.D., Liang, C., et al. (2013) Study on Psychological Factors and Psychological Intervention in Patients with Recurrent Abortion. China Health Education, 29, 843-845. 
[3] Xu, B., Wang, W.D. and Liu, S.L. (2000) Psychosomatic Medicine-Fundamentals and Clinics of Psychophysiological Medicine. China Science and Technology Press, Beijing, 148-158.

[4] Chen, Q.R. (2011) Study on the Social and Psychological Factors of Recurrent Abortion. Chinese Journal of Maternal and Child Health, 26, 31-32.

[5] Yan, Y.L., Wan, B., Hu, W.J., et al. (2011) Clinical Analysis of Psychological Stress in Recurrent Abortion. Clinical Practice, 12, 70-76.

[6] Wang, L.L. and Xu, K.H. (2012) Study on Quality of Life and Its Influencing Factors in Patients with Recurrent Miscarriage. Sichuan Medicine, 33, 615-617.

[7] Wu, J.H., Wei, L.F., Lin, W.X., et al. (2012) The Effect of Immunotherapy on Nursing Care of Patients with Recurrent Abortion. Journal of Nursing. Surgical Edition, 27, 39-40.

[8] Feng, Y. (2008) Obstetrics Gynecology. People’s Medical Publishing House, Beijing, 64-67.

[9] Cao, F.L., Zhang, Y.Z., Gao, Q.L., et al. (2004) The Relationship between Mental Health Status and Coping Style in Patients with Recurrent Spontaneous Abortion. Journal of Nursing, 19, 12-14.

[10] Duan, C.Y. and Fan, L. (2007) The Effect of Marital Quality and Family Function on Pregnant Women's Psychology. China Maternal and Child Health, 22, 3374-3375.

[11] Chai, J. (2010) The Prognosis of Early Threatened Abortion and the Effect of Various Treatments. Chinese Medical Guideline, No. 35, 695-696.

[12] Cao, Q.Y. (2006) Mental Health Problems of Elderly Women and Women. Journal of Applied Gynecology and Obstetrics, 23, 729-732.

[13] Cai, J.H. (2011) Effect of Psychological Intervention on the Treatment of Recurrent Abortion with Unknown Cause. General Nursing, 9, 2370-2371.

[14] Wu, Y.P., Chen, B.J. and Huang, S.T. (2010) Analysis of Psychological Factors and Countermeasures of Pregnant Women with Recurrent Abortion. Inner Mongolia Journal of Traditional Chinese Medicine, 29, 164-166.

[15] Practice Committee of the American Society for Reproductive Medicine (2012) Evaluation and Treatment of Recurrent Pregnancy Loss: A Committee Opinion. Fertility and Sterility, 98, 294-301.

[16] Kaandorp, S.P., Goddijn, M., van der Post, J.A., et al. (2010) Aspirin plus Heparin or Aspirin Alone in Women with Recurrent Pregnancy Loss. The New England Journal of Medicine, 362, 1586-1596. https://doi.org/10.1056/NEJMoa1000641

[17] Jauniaux, E., Farquharson, R.G., Christiansen, O.B., et al. (2006) Evidence-Based Guidelines for the Investigation and Medical Treatment of Recurrent Miscarriage. Human Reproduction, 21, 2216-2222. https://doi.org/10.1093/humrep/del150

[18] Du, J.P. (2011) Study on the Relationship between Family Function and Mental Health Status of Pregnant Women. Chinese Foreign Health Abstracts, 8, 41-42. (In Chinese)

[19] Liu, H., Li, G.P., Zhang, L.Y., et al. (2008) The Effect of Family Function on Mental Health and Treatment Attitude of Infertile Women. China Maternal and Child Health, 23, 3566-3567.

[20] Yang, L. (2011) Study on the Physique of Women with Recurrent Abortion. Shandong University of Traditional Chinese Medicine, Jinan. 